\title{
An Optimized Measurement Algorithm for Gas Sensors Based on Carbon Nanotubes: Optimizing Sensor Performance and Hardware Resources
}

\author{
Encarnación Castillo, José F. Salmerón, Aniello Falco, Florin C. Loghin, Francisco J. Romero, \\ Paolo Lugli, Diego P. Morales ${ }^{(0)}$, and Almudena Rivadeneyra ${ }^{(0)}$
}

\begin{abstract}
This paper presents a novel algorithm for the 2 measurement of resistive-type gas sensors with carbon nan3 otubes (CNTs) as sensitive layer. Contrary to conventional 4 strategies, which extract the sensor information from the normal5 ized resistance, the proposed algorithm is based on the variation 6 in resistance over time. The results have demonstrated that the 7 time necessary to get the maximum performance of these sensors 8 is reduced around a $25 \%$ when compared with the conventional 9 approach for any of the recovery strategies analyzed (passive 0 desorption, external heating, or dc voltage). The hardware imple1 mentation of the proposed algorithm in a field-programmable 12 gate array (FPGA) has also demonstrated that, in addition to 13 optimizing the sensor performance in terms of time response 14 and sensitivity, this measurement algorithm yields a significant 5 minimization of the sensor readout circuit resources at both soft6 ware and hardware levels paving the way for future development of smart sensors for the Internet of Things (IoT) applications.
\end{abstract}

Index Terms-Carbon nanotube (CNT), field-programmable 9 gate array (FPGA), inkjet printing, $\mathbf{N H}_{3}$, recovery, single-walled, 20 spray deposition.

21

${ }_{22}$ ECENTLY, gas leakage detection is a topic of high 23 interest in diverse fields from personal to industrial appli24 cations. In this context, thanks to the rapid advances in the 25 development of wireless sensors devices into the Internet of 26 Things (IoT) paradigm, there is a current trend toward the 27 ubiquitous sensing. However, significant challenges remain 28 concerning the widespread availability of this kind of devices 29 for end-user applications. On the one hand, sensors need to so be lightweight, low-power, robust, and small in size and, on ${ }_{31}$ the other hand, the algorithms implemented in the IoT devices 32 must be optimized to minimize both computing resources and 33 time, and consequently, the power consumption.

Manuscript received March 18, 2019; revised May 17, 2019 and June 18 2019; accepted July 6, 2019. (Corresponding author: Almudena Rivadeneyra.)

E. Castillo, F. J. Romero, D. P. Morales, and A. Rivadeneyra are with the Department of Electronics and Computer Technology, University of Granada, 18071 Granada, Spain (e-mail: encas@ugr.es; franromero@ugr.es; diegopm@ugr.es; arivadeneyra@ugr.es).

J. F. Salmerón and F. C. Loghin are with the Institute for Nanoelectronics, Technical University of Munich, 80333 Munich, Germany (e-mail: jf.salmeron@tum.de; florin.loghin@tum.de).

A. Falco and P. Lugli are with the Faculty of Science and Technology, Free University of Bozen-Bolzano, 39100 Bolzano, Italy (e-mail: aniello.falco@unibz.it; paolo.lugli@unibz.it).

Digital Object Identifier 10.1109/JIOT.2019.2928231
Therefore, it is clear that the convergence between nanotechnology (new materials and detection methods, manufacturing 35 processes, device integration, etc.) and both measuring and 36 processing algorithms is mandatory to move forward in this ${ }_{37}$ effort [1].

Regarding gas sensors, resistive ones are very popular 39 because of their reasonable price and good durability [2]. 40 However, they are commonly limited in their selectivity and 41 sensitivity, boosting research to develop new materials and 42 detection methods for a better gas discrimination [3]. In this direction, functional materials have received a lot of attention. A large variety of electrical sensor materials have been investigated, including semiconducting metal oxides, silicon devices, organic materials, carbon nanotubes (CNTs) and carbon black-polymer composites [4]. These new materials are normally tuned by functionalizing them with metal and polymers to achieve selectivity toward certain gas species [5]; however, the vast majority of the solutions are time and power demanding. Therefore, several approaches have been developed toward facing these issues, for instance, higherorder sensing systems which consist of sensor arrays covered with different sensitive layers [6], using pattern recognition algorithms [7], or multifunctional sensors which measure different properties of a sensitive layer [8]. Furthermore, it is desirable to recover the sensor response over time, that is to say, to desorb the trapped molecules in the sensing element after being exposed to the gas. A common strategy to recover the sensing layer is to externally heat the sensor to increase the desorption rate of the trapped molecules [9]-[11]. This approach normally requires the integration of more circuitry and higher power consumption. Recently, we have reported 64 the capability of CNTs gas sensors to self-recover by apply- 65 ing a higher than probing dc voltage between its terminals, 66 reducing the power demand of the system as well as not ${ }_{67}$ requiring more circuit elements for the recovery stage [12]. 68 A lot of effort has been put in developing this kind of gas 69 sensor because they present high sensitivity toward a vari- 70 ety of gas species [5], [13], [14], can be manufactured with 71 cost-effective techniques [15], [16] and can be deposited on 72 flexible substrates [17], [18], providing a promising starting 73 point for the industrial use of this technology. However, very 74 limited attention has been paid to their transduction mecha- 75 nisms in terms of the requirements to be integrated at system 76 level.

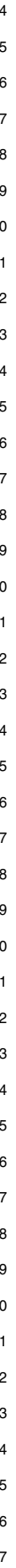

2327-4662 (c) 2019 IEEE. Personal use is permitted, but republication/redistribution requires IEEE permission. See http://www.ieee.org/publications_standards/publications/rights/index.html for more information. 
78 Conventionally, the information of resistive sensors is 79 extracted from their normalized resistance (NR). In other 80 words, it is measured the relative change in resistance when 81 the sensor is exposed to the parameter of interest with respect 82 to its value under base level conditions [10], [19], [20]. One 83 essential part of any sensory system, mostly neglected by basic ${ }_{84}$ research, is the integration of the sensing element in a com85 plete electronic system. This requires the design of circuits to 86 adapt the sensor signal and efficiently and accurately extract 87 the sensor information [21].

${ }_{88}$ In this paper, we propose a new algorithm to analyze the 89 response of a CNT gas sensor based on its relative change 90 of resistance over time and we demonstrate its superior91 ity over the NR, the conventional approach, at the sensor 92 performance and at the employed hardware resources for 93 extracting information. We investigate this novel approach in 94 terms of time response, sensitivity, and level of integration 95 in final systems. One of the most desirable aspects in the 96 field of instrumentation is to bring the intelligence to the sen97 sor elements through the applications of low computational 98 cost, small size, and low consumption. Thus, the measure99 ment algorithm proposed in this paper allows optimizing the 100 resources of implementation and computation, making feasi101 ble the future development of a smart sensor, crucial for the 102 truly development of the paradigm of the IoT.

103 This paper is organized as follows. In Section II, the design 104 and fabrication of the sensor is described together with its 105 characterization procedure. Section III describes the proposed 106 algorithm and presents its empirical results in comparison 107 with the conventional method. At the end of this section, 108 it is analyzed the system requirements of each approach 109 and their feasibility of circuitry integration. Finally, the main 110 conclusions are drawn in Section IV.

\section{Materials And Methods}

\section{A. Fabrication Process}

113 Initially, we sprayed the CNT solution on the polyimide 114 substrate (Kapton HN) of $125 \mu$ m-thickness. The CNTs were 115 dispersed in an aqueous solution based on $1 \mathrm{wt} \%$ sodium 116 dodecyl sulfate (SDS). After stirring the solution for $1 \mathrm{~h}$, 1170.03 wt.\% CNTs were added to the dissolved dispersion agent. 118 The obtained solution was sonicated for $30 \mathrm{~min}$ using horn 119 sonicator at $50 \%$ of its maximum power $(\sim 48 \mathrm{~W})$. After $1.5 \mathrm{~h}$ 120 of centrifugation at $15000 \mathrm{rpm}$, the top part of the solutions 121 was removed from the vials to be used for the deposition, while 122 the bottom $20 \%$ was disposed. By mounting an air atomizing 123 spray valve (Nordson EFD, USA), to a motorized $X-Y$ plat124 form (Precision Valve \& Automation, USA), it was possible 125 to obtain a repeatable spray process, using an approach similar 126 to what previously reported [17], [18]. After deposition, the 127 samples were immersed in deionized water for $15 \mathrm{~min}$ at room 128 temperature to remove the dispersant, and subsequently dried. 129 Then, on top of the CNT film, we defined the electrodes, 130 using a DMP-2831 Dimatix printer (Fujifilm Dimatix, Inc., ${ }_{131}$ Santa Clara, CA, USA) fixing the plate temperature during the 132 printing at $60{ }^{\circ} \mathrm{C}$. The electrodes were made of one layer of sil${ }_{133}$ ver nanoparticles (DGP 40LT-15C from ANP Company, USA),

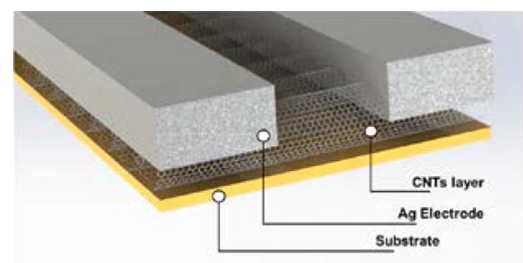

(a)

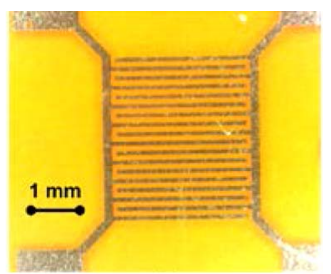

(b)

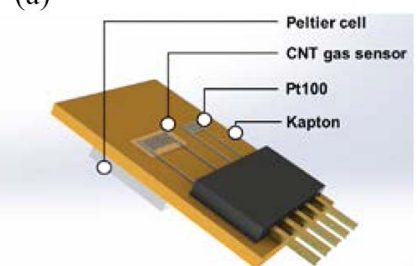

(c)

Fig. 1. (a) Schematics of the fabricated sensors. (b) Real picture of the sensor. (c) Schematic of sensor module.

defining the drop space to $50 \mu \mathrm{m}$. The finger width and the 134 space between fingers are $100 \mu \mathrm{m}$ and the finger length $3 \mathrm{~mm} . \quad{ }_{135}$ After printing and drying of the electrodes, they were photonic 136 sintered (Sinteron 2010 from Xenon, USA) with five pulses 137 of $2.5 \mathrm{kV}$ energy and $500 \mu \mathrm{s}$ width. Fig. 1(a) illustrates the 138 layers of the manufactured device and Fig. 1(b) presents a real 139 picture of the developed sensor.

The complete sensor module includes the resistive sensor, 141 mounted on a carrier glass together with a Peltier heating 142 element for temperature control and a temperature sensor 143 (Pt100) for in-situ monitoring [Fig. 1(c)].

\section{B. Characterization Process}

Sensors were measured by monitoring the variation in resis- 146 tance with respect to the test gas concentration. Before the ${ }_{147}$ sensor response toward test gas was analyzed, the initial resis- 148 tance of the sensor was monitored over time to determine its 149 baseline. After reaching a stable initial value, the sensor mod- 150 ule was introduced inside a gas chamber and its response was 151 evaluated by exposing it to different concentrations of test gas. 152 Fig. 2 illustrates a measurement cycle, which is composed of 153 an exposure interval followed by a recovery interval and then 154 an interval at normal conditions. The room temperature was 155 set to circa $22^{\circ}$, and in each stage, nitrogen was employed 156 as carrier gas to be certain that the measurement result would 157 only be influenced by the target gas. In "normal conditions" 158 phases, the carrier gas flow was set to $200 \mathrm{ml} / \mathrm{min}$. During 159 exposure phases, the overall mixture flux was set to a constant 160 $200 \mathrm{ml} / \mathrm{min}$ for a given time at ambient temperature [stage (c) 161 in Fig. 2].

162

Recovery was then introduced, following three different 163 strategies [stage (b) in Fig. 2]: 1) externally heating the sen- 164 sor, with a Peltier cell, to $80{ }^{\circ} \mathrm{C}$; 2) applying $10 \mathrm{~V}$ among 165 the sensor terminals to establish a self-heating condition; and 166 3) passive recovery (leaving the sensor under ambient condi- 167 tions). For ease of comparison, every recovery strategy was 168 tested with the same conditions (unchanged room tempera- 169 ture, $200 \mathrm{ml} / \mathrm{min}$ flux of carrier gas). After these recovery 170 


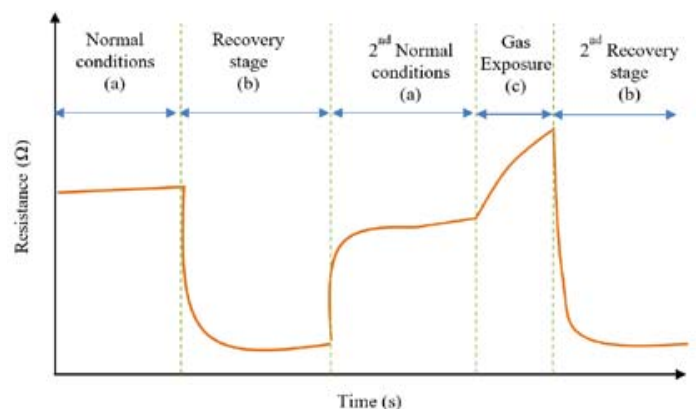

Fig. 2. Schematic of the measurement flow, pointing out the different stages. (a) Operation at normal conditions. (b) Recovery step. (c) Exposure to gas.

171 phases, the high flux was kept once more at ambient condi172 tions [stage (a) in Fig. 2] to facilitate the recuperation of the 173 device and purge any residual test gas molecules out of the gas 174 chamber. Recovery stage ended by a final step under sensing 175 conditions ( $200 \mathrm{ml} / \mathrm{min}$ of carrier gas at ambient conditions) 176 to help restore the initial resistance before the next expo177 sure cycle. We performed two different measurement tests, 178 a longer one where all the steps in the cycle had a duration 179 of $300 \mathrm{~s}$ and a shorter one with the duration of the steps 180 fixed at $150 \mathrm{~s}$.

181 The measurements were automated with LabVIEW 1822016 software, which controlled an impedance analyzer ${ }_{183}$ (Keysight E4990A) with an impedance probe kit (4294A1) for 184 the sensor readout (impedance). The excitation voltage applied 185 in all measurements was $V_{\mathrm{dc}}=0$ and $V_{\mathrm{ac}}=500 \mathrm{mV}$ and 186 the frequency swept ranged from 20 to $100 \mathrm{~Hz}$. The dc volt187 age to perform the recovery stage was directly applied by the 188 impedance analyzer. The phase in all frequencies was below $18910^{-30}$, proving the virtually pure resistive behavior of the 190 device under test. In particular, the $\mathrm{NH}_{3}$ concentrations tested 191 covered a range between 10 and 80 ppm, achieved by diluting 192 the test gas with nitrogen as carrier gas.

\section{RESULTS AND DISCUSSION}

194 In this section, we will first show the analysis of the 195 described sensor using the proposed measurement methods 196 and comparing its performance with the conventional proce197 dure. After that, we will show the influence of the recovery 198 strategy in this new measurement protocol, followed by the 199 use of this protocol to the response of the sensor toward 200 other gases. Finally, we will show a study of the efficiency 201 of the described measurement protocol in comparison with 202 the conventional one in hardware and software final solutions. 203 In the following sections, we present the mean values of 204 three complete measurements cycles. The errors are lower than $2052 \%$ in all cases.

\section{A. Response to Ammonia}

207 In order to evaluate the sensor performance, the conven208 tional measurement protocol is based on the NR presented in 209 (1): the difference in resistance before and after the exposure 210 divided by this initial resistance. What we propose is to fix the 211 measurement time and only measure the variation in resistance in the chosen time slot, see (2)

$$
\begin{aligned}
\text { Normalized Resistance }(\%) & =\frac{R_{f}-R_{i}}{R_{i}} \quad \text { (1) } 213 \\
\text { Temporal Resistance Variation }(\Omega / s) & =\frac{R_{f}-R_{i}}{T} \quad \text { (2) } 214
\end{aligned}
$$

where $R_{i}$ and $R_{f}$ are the initial and final resistance values of 215 an exposure cycle, respectively, and $T$ is the reference time 216 interval. Notice that once that after the calibration, an optimal 217 value of $T$ will be found, it shall be considered as constant. 218 On the contrary, looking to (1), the initial resistance of the 219 CNT film cannot be considered as constant, since the baseline 220 value is not necessarily recovered after exposure.

221

The time response of such gas sensing systems is tradition- 222 ally modeled as a "charging" circuit, with a classic growing 223 negative exponential response [22]

$$
\begin{aligned}
R(t) & =R_{i}+\Delta R(C)\left(1-e^{-\frac{t}{\tau}}\right) \\
R^{\prime}(t) & =\frac{\Delta R(C)}{\tau} e^{-\frac{t}{\tau}} \\
N R^{\prime} & =\frac{\Delta R(C)}{R_{i} \tau} e^{-\frac{t}{\tau}} .
\end{aligned}
$$

The derivative of the time evolution of resistance-and, 228 hence, the NR-after exposure is always strictly positive, as 229 presented in (4) and (6), respectively. This implies that the 230 NR will have an increasing value until the slope will be 231 only marginally increased, and saturation can be considered 232 reached. The saturation point, however, could be substantially 233 different, depending on the network properties. The proposed 234 characterization feature, on the other hand, focuses on the tem- 235 poral resistance variation (TRV), introduced in the previous 236 section. The TRV will quickly reach its maximum and then 237 decrease over time, toward a more stable value. Proceeding 238 similar to what we did for the NR, it is possible to write TRV 239 and its derivative as

$$
\begin{aligned}
\operatorname{TRV}(t) & =\frac{\Delta R(C)}{t}\left(1-e^{-\frac{t}{\tau}}\right) \\
\operatorname{TRV}^{\prime}(t) & =-\frac{\Delta R(C)}{t^{2}}\left(1-e^{-\frac{t}{\tau}}\right)+\frac{\Delta R(C)}{t \tau} e^{-\frac{t}{\tau}} .
\end{aligned}
$$

Thus, the derivative with respect to time of TRV is strictly 243 negative and quickly approaching zero. The maximum of the 244 TRV response will be observed immediately after exposure. 245

Fig. 3(a) and (b) shows the NR while the sensor is exposed 246 to the test gas, whereas Fig. 3(c) and (d) presents the TRV ${ }_{247}$ during the test gas exposure. In particular, Fig. 3(a) and (c) 248 corresponds to $150 \mathrm{~s}$ exposure while Fig. 3(b) and (d) to $300 \mathrm{~s} .249$ It can be observed how the shapes of the curves are indepen- 250 dent on the exposure time. In the case of the NR, the sensor 251 has not achieved the saturation point. If the exposure takes 252 longer, it is expected to have an increase in the NR and, there- 253 fore, the response of the sensor would be higher, the longer 254 the exposure is. Contrary to this behavior, the TRV depicts 255 a faster increase in its response around the first $50 \mathrm{~s}$ exposure 256 and it tends to a constant value above $120 \mathrm{~s}$. This response 257 provides us a new resource to characterize this sensor, ensur- 258 ing a stable value and, especially, facilitating its integration in 259 final systems. 


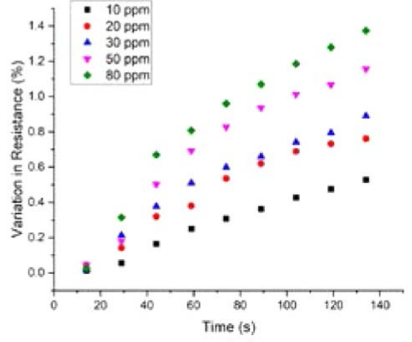

(a)

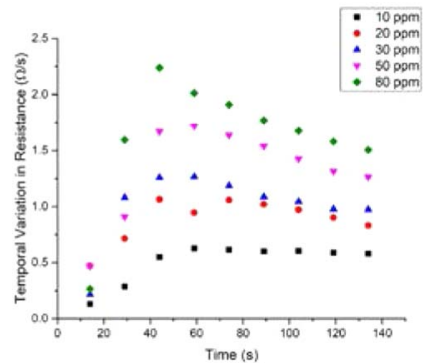

(c)

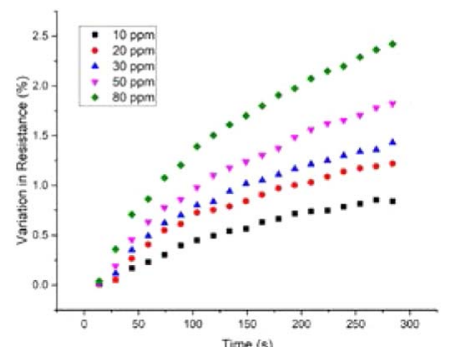

(b)

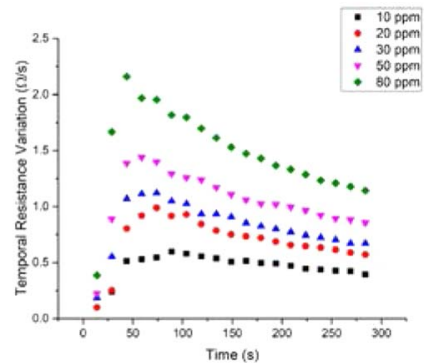

(d)
Fig. 3. NR over time for different $\mathrm{NH}_{3}$ concentrations with an exposure time of (a) $150 \mathrm{~s}$ and (b) $300 \mathrm{~s}$. TRV over time at the same $\mathrm{NH}_{3}$ concentrations with an exposure time of (c) $150 \mathrm{~s}$ and (d) $300 \mathrm{~s}$.

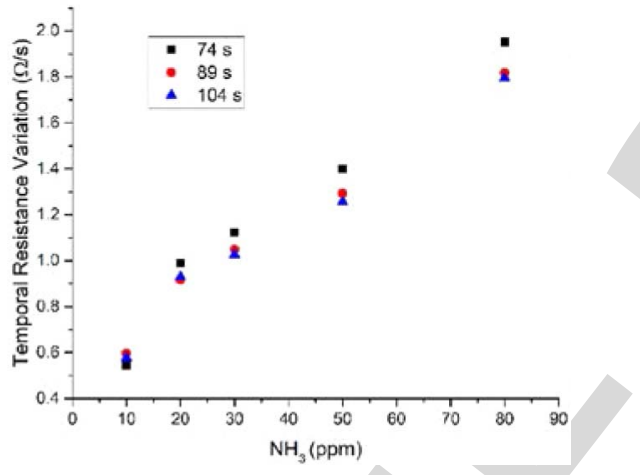

Fig. 4. Temporal resistance variation versus ammonia concentration at different exposure times.

261 The calibration curve of the sensor considering the TRV 262 over $\mathrm{NH}_{3}$ concentration is illustrated in Fig. 4, selecting 263 three different point of time. At $74 \mathrm{~s}$, the response is slightly 264 higher than at longer time exposure. Contrary to the traditional 265 measurement (NR), longer exposure time does not ensure 266 a better response of the sensor.

267 Basically, when using NR, the response achieves its maxi268 mum at saturation and to reach this point we have to wait until 269 the resistance stabilizes (meaning in this case, a time longer 270 than $300 \mathrm{~s}$ ). Opposite to this approach, when applying TRV, 271 how fast the resistance changes with the concentration exhibits 272 an increase at the beginning until reach a maximum (in this 273 case, about 75 s) and then stabilized.

274 The linear regression curves extracted from data in Fig. 4 are 275 presented in (8)-(10) for 74, 89, and $104 \mathrm{~s}$, respectively

${ }_{276} \operatorname{TVR}(\Omega / s)=0.01844 \cdot\left[\mathrm{NH}_{3}\right]+0.50105, R^{2}=0.95138$

${ }_{277} \operatorname{TVR}(\Omega / s)=0.01631 \cdot\left[\mathrm{NH}_{3}\right]+0.51455, R^{2}=0.97501$

$278 \operatorname{TVR}(\Omega / s)=0.01603 \cdot\left[\mathrm{NH}_{3}\right]+0.50833, R^{2}=0.96319$.

279
Notice an important improvement that TRV achieves com- 280 pared to the NR for the integration of the sensing element in 281 a complete electronic system. For the computation of the NR 282 it can be observed that (1) includes a division to an arbitrary 283 real number (the measured initial resistance value). This arith- 284 metic operation, albeit important, is far more complex than 285 addition and multiplication, and requires significant amount of 286 resources for a complete hardware implementation [23], [24]. 287 Thus, a challenge, sometimes difficult to achieve, is to be able 288 to manipulate the algebraic expression in order to convert it 289 into another one that only includes multiplications and/or addi- 290 tions. Achieving the above would make it possible to get an 291 area reduction and performance improvement of the hardware 292 implementation, a very important aspect to be able to provide 293 a competitive portable design. In this sense, for the computa- 294 tion of the TRV, (2) matches the requirements of the previous 295 challenge, since the term $T$ is a constant value, statically cho- 296 sen in design phase, and the division by this constant can be 297 easily converted into a multiplication [25]. Thus, in addition 298 to the advantage related to the faster increase in its response, 299 the TRV facilities the integration in final systems. 300

Similar results have been found for ethanol (see Fig. S1), 301 demonstrating the wider use of the proposed algorithm. 302

Muezzinoglu [26] also proposed an analysis of the tran- 303 sient response of chemo-resistive sensors providing a faster 304 response without waiting until the sensor achieve the equilib- 305 rium point. However, in their approach, it is needed to employ 306 various multiplications; while, in our algorithm, only one sim- 307 ple multiplication is needed. Moreover, contrary to them, we 308 demonstrate that no data transformation is needed to extract 309 the sensor information.

310

\section{B. Response Using Different Recovery Strategies}

311

The recovery of the sensor to its baseline after finishing the 312 exposure to the gas is an important feature of any gas sen- 313 sor, although the vast majority does not completely recover 314 to their baseline but they show substantial drifts after differ- 315 ent exposure cycles [27], [28]. SWNT gas sensors are one of 316 those devices that do not recuperate the baseline at ambient 317 conditions after exposure to a test gas [29]. In order to force 318 the sensor to recover its initial characteristics, it is necessary 319 to provide extra energy in the form of heat or gate biasing or 320 UV light [29]-[32]. The data presented in Figs. 3 and 4 cor- 321 responds to a recovery strategy based on external heating (in 322 this case, at $80{ }^{\circ} \mathrm{C}$ ), as this is the most established approach. 323 Nevertheless, recently it has been proved that a similar effect ${ }_{324}$ can be achieved by applying a dc voltage [12]. Even in this 325 case, however, the power demand of the sensing system is sig- 326 nificantly higher than what a simple resistance measurement 327 would require. Resultantly, an even more remarkable finding 328 would be to avoid the recovery to the baseline, leaving the 329 sensor to passively get rid of the trapped molecules (passive 330 recovery), and still be able to guarantee high and repeatable 331 response.

Fig. 5 shows the analysis presented in the previous section 333 for dc voltage recovery [Fig. 5(a) and (b)] and for passive 334 recovery [Fig. 5(c) and (d)]. The shapes obtained are similar 335 


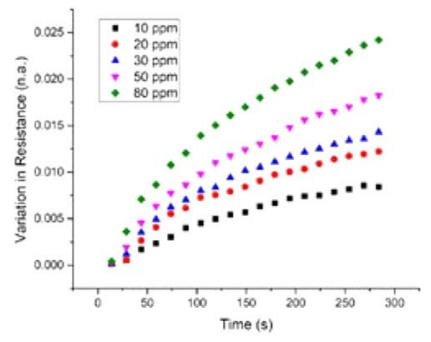

(a)

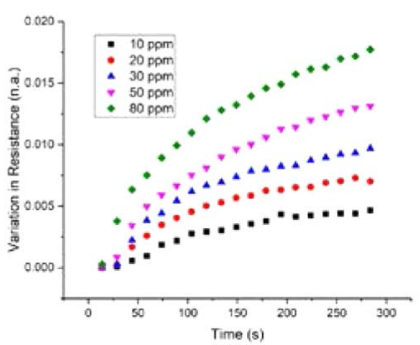

(c)

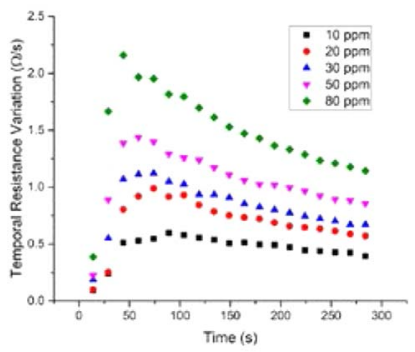

(b)

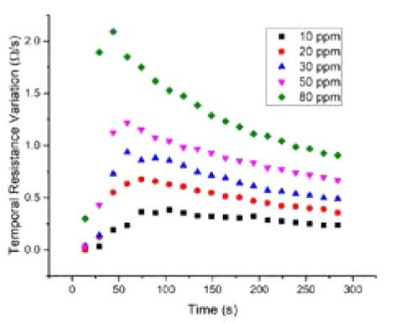

(d)
Fig. 5. (a) $\mathrm{NR}$ over time for different $\mathrm{NH}_{3}$ concentrations with an exposure time of $300 \mathrm{~s}$ and recovery at $10 \mathrm{~V}$. (b) TRV over time at the same $\mathrm{NH}_{3}$ concentrations with an exposure time of $300 \mathrm{~s}$ and recovery at $10 \mathrm{~V}$. (c) NR over time for different $\mathrm{NH}_{3}$ concentrations with an exposure time of $300 \mathrm{~s}$ and passive recovery. (d) TRV over time at the same $\mathrm{NH}_{3}$ concentrations with an exposure time of $300 \mathrm{~s}$ and passive recovery.

336 to the external heating recovery for both NR and TRV. When 337 we look at the NR, the active recovery strategies (heating at $33880{ }^{\circ} \mathrm{C}$ and applying $10 \mathrm{~V}$ ) achieve around $2.5 \%$ at $80 \mathrm{ppm}$ $339 \mathrm{NH}_{3}$ after $300 \mathrm{~s}$, while the response is below $2 \%$ for passive 340 recovery at the same concentration and point of time. But when ${ }_{341}$ we look at the TRV, this difference is reduced. For example, 342 we measured $1.9 \Omega / \mathrm{s}$ at $80 \mathrm{ppm} \mathrm{NH}_{3}$ and $74 \mathrm{~s}$ for heating 343 recovery, $2.0 \Omega / \mathrm{s}$ for dc voltage recovery, and $1.8 \Omega / \mathrm{s}$ for 344 passive recovery. Hence, the response loss with respect to the 345 active recovery case is $18 \%$ and $5 \%$ for the NR and TRV 346 methods, respectively.

\section{${ }_{347}$ C. Performance in Real Measurement Instrument}

348 Hardware implementation in field-programmable gate 349 array (FPGA) of NR and the new proposal based on 350 TRV have been evaluated and compared. Fig. 6 shows 351 the block diagram of TRV implementation that is based 352 on a finite-state machine (FSM) implementation. VHDL 353 descriptions of the developed designs have been implemented 354 using Spartan 6 xc6vlx40t-1fgg1156 [33] and Cyclone II 355 EP2C20F484C7 [34] devices from Xilinx and Intel (formerly, 356 Altera), respectively. The architectures have been designed for 357 two different exposure times, 128 and $256 \mathrm{~s}$. These exposure 358 times are within the range of interest and have been selected 359 since to divide by a power of 2 using binary representation is 360 equivalent to displacements to the right of the data record, 361 thus reducing the required area resources. In order to get 362 these exposure times, the designs were developed taking into 363 account the frequencies of the clock sources coming into the ${ }_{364}$ FPGA devices. 200 and $50 \mathrm{MHz}$ clock sources were used for ${ }_{365}$ Spartan 6 and Cyclone II devices, respectively.

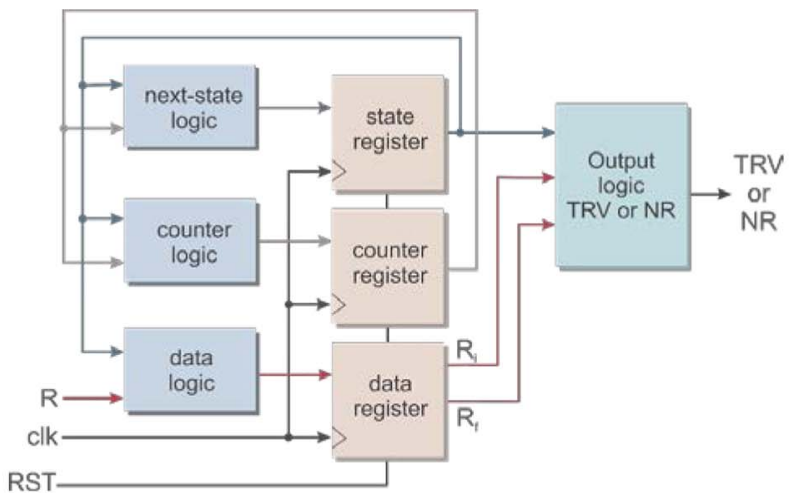

(a)

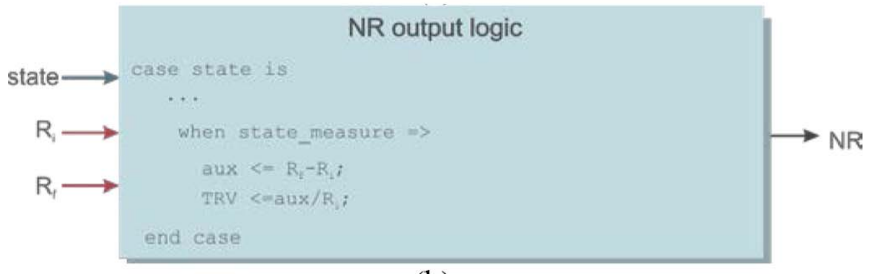

(b)

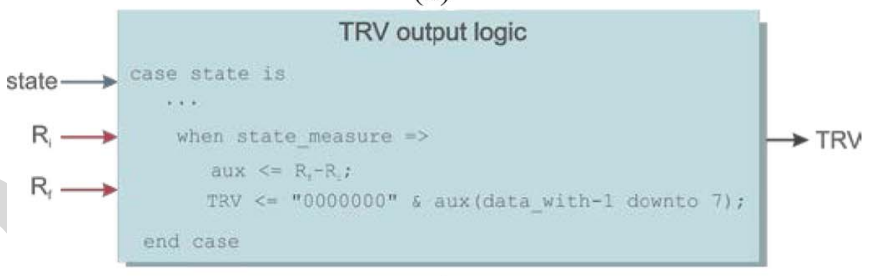

(c)

Fig. 6. (a) Block diagram of TRV or NR algorithm implemented in FPGA. (b) VHDL sentences for NR output logic for NR algorithm. (c) VHDL sentences for TRV output logic (for an exposure time of $128 \mathrm{~s}$ ).

TABLE I

AREA AND DELAy FIgURES FOR FPGA IMPLEMENTATION USING AN EXPOSURE TIME OF $128 \mathrm{~S}$

\begin{tabular}{|c|c|c|c|c|}
\hline \multirow[b]{2}{*}{ Design } & \multicolumn{4}{|c|}{$\begin{array}{l}\text { Spartan } 6 \\
\end{array}$} \\
\hline & $\begin{array}{c}\text { Area } \\
\text { (Slices) }\end{array}$ & Ratio & $\begin{array}{c}\mathbf{F}_{\max } \\
(\mathbf{M H z})\end{array}$ & Ratio \\
\hline NR & 99 & 1 & 29.49 & 1 \\
\hline TRV & 25 & 0.25 & 478.87 & 0.06 \\
\hline \multirow[b]{2}{*}{ Design } & \multicolumn{4}{|c|}{ "Cyclone II } \\
\hline & $\begin{array}{l}\text { Area } \\
\text { (LEs) } \\
\end{array}$ & Ratio & $\begin{array}{c}\mathbf{F}_{\max } \\
(\mathbf{M H z})\end{array}$ & Ratio \\
\hline NR & 374 & 1 & 20.27 & 1 \\
\hline TRV & 83 & 0.22 & 189.04 & 0.11 \\
\hline
\end{tabular}

Tables I and II summarize the area and maximum frequency 366 figures for these FPGA implementations. It can be observed 367 from these tables that TRV design occupies a much smaller 368 area than NR design. Concretely, TRV design occupies 369 around $75 \%$ less area than NR for both exposure times and 370 FPGA devices. In addition to this, the high surface and the 371 more complex structures lower significantly the operating 372 frequency of the circuits. These tables also show how the 373 NR designs in the Spartan 6 and Cyclone II fall short of 374 the reference frequencies of 200 and $50 \mathrm{MHz}$. For instance, 375 in case of the Spartan 6, the maximum frequency stops at 376 
TABLE II

AREA AND DELAY FIgURES FOR FPGA IMPLEMENTATION USING AN EXPOSURE TIME OF $256 \mathrm{~s}$

\begin{tabular}{ccc||cc}
\hline \hline \multirow{2}{*}{ Design } & \multicolumn{4}{c}{ Spartan 6 } \\
\cline { 2 - 5 } & $\begin{array}{c}\text { Area } \\
\text { (Slices) }\end{array}$ & Ratio & $\begin{array}{c}\mathbf{F}_{\max } \\
(\mathbf{M H z})\end{array}$ & Ratio \\
\hline \hline NR & 101 & 1 & 30.25 & 1 \\
TRV & 27 & 0.27 & 327.44 & 0.09 \\
\hline \hline \multirow{3}{*}{ Design } & \multicolumn{4}{c}{ Cyclone II } \\
& Area & Ratio & $\begin{array}{c}\text { F }_{\max } \\
\text { (MHz) }\end{array}$ & Ratio \\
\hline \hline NR & 377 & 1 & 20.07 & 1 \\
TRV & 86 & 0.23 & 199.20 & 0.11 \\
\hline \hline
\end{tabular}

37729.29 and $30.25 \mathrm{MHz}$ for an exposure time of 128 and $256 \mathrm{~s}$, 378 respectively. A similar situation occurs for Cyclone II devices 379 and NR designs. To effectively use these circuits, a clock 380 divider component should be implemented, increasing thus 381 the area resources. In this sense, TRV designs substantially 382 improve the maximum frequency, increasing around a $90 \%$ the 383 maximum frequencies. For example, the maximum frequencies 384 supported by TRV designs for Spartan 6 device are 478.87 and $385327.44 \mathrm{MHz}$ for an exposure time of 128 and $256 \mathrm{~s}$, respec386 tively, which satisfy the required frequency of $200 \mathrm{MHz}$. As 387 a result, TRV designs in addition to reduce area occupation, 388 allow reaching the required frequencies, without being nec389 essary to include a clock divider component, which further 390 increase the complexity.

\section{CONCLUSION}

392 This paper proposes and develops a novel measurement 393 algorithm for gas sensors based on single-walled CNTs. This 394 algorithm not only optimizes the sensor performance in terms 395 of time response and sensitivity but also facilitates the inte396 gration of the sensor in a final system, minimizing hardware 397 resources, and power consumption.

398 The described algorithm is based on the measurement of 399 the variation in resistance over time, what we define as time 400 resistance variation (TRV). We demonstrate that only $74 \mathrm{~s}$ 401 are necessary to get the maximum performance of the sensor 402 response for ammonia detection and this value is applicable for 403 different recovery strategies (passive desorption, external heat$404 \mathrm{ing}$, and dc voltage). In the case of the conventional approach, 405 measuring the NR does not get the best performance below $406300 \mathrm{~s}$ for any of the recovery strategies analyzed. Although 407 both calculations of TRV and NR involve a division, the ${ }_{408}$ practical computation of TRV can be reduced by a subtrac409 tion of two resistance values whereas the division in NR 410 cannot be simplified, because the initial resistance changes 411 over time. Therefore, from a computational point of view and 412 in terms of circuit resources, TRV results in a faster solu413 tion, requiring a minimal number of electronic blocks for the 414 hardware implementation to extract the sensor information. ${ }_{415}$ Thus, the hardware implementation in FPGA of NR and ${ }_{416}$ TRV designs has demonstrated that TRV design reduces 417 the area resources and increases substantially the operating 418 frequency.

\section{REFERENCES}

[1] F. V. Paulovich, M. C. F. De Oliveira, and O. N. Oliveira, "A future 420 with ubiquitous sensing and intelligent systems," ACS Sensors, vol. 3, 421 no. 8, pp. 1433-1438, Aug. 2018.

[2] G. Sberveglieri, "Recent developments in semiconducting thin-film gas 423 sensors," Sensors Actuators B Chem., vol. 23, nos. 2-3, pp. 103-109, 424 Feb. 1995.

[3] J. M. Smulko et al., "New approaches for improving selectivity and 426 sensitivity of resistive gas sensors: A review," Sensor Rev., vol. 35, no. 4, 427 pp. 340-347, Apr. 2015.

[4] K. Arshak, E. Moore, G. M. Lyons, J. Harris, and S. Clifford, "A review 429 of gas sensors employed in electronic nose applications," Sensor Rev., 430 vol. 24 , no. 2, pp. 181-198, 2004.

[5] K. Balasubramanian and M. Burghard, "Chemically functionalized 432 carbon nanotubes," Small, vol. 1, no. 2, pp. 180-192, Dec. 2004.

[6] C. Hagleitner et al., "Smart single-chip gas sensor microsystem," Nature, 434 vol. 414, pp. 293-296, Nov. 2001.

17] F. Hossein-Babaei, S. M. Hosseini-Golgoo, and A. Amini, "Extracting 436 discriminative information from the Padé-Z-transformed responses of 437 a temperature-modulated chemoresistive sensor for gas recognition," 438 Sensors Actuators B Chem., vol. 142, no. 1, pp. 19-27, Oct. 2009.

[8] J. A. Covington, S. L. Tan, J. W. Gardner, A. Hamilton, T. Koickal, and 440 T. Pearce, "Combined smart chemFET/resistive sensor array," in Proc. 441 IEEE SENSORS, 2003, pp. 1120-1123.

[9] D. Vincenzi, "Development of a low-power thick-film gas sensor 443 deposited by screen-printing technique onto a micromachined hotplate," 444 Sensors Actuators B Chem., vol. 77, nos. 1-2, pp. 95-99, Jun. 2001. 445

[10] K. H. Crowley et al., "Fabrication of an ammonia gas sensor 446 using inkjet-printed polyaniline nanoparticles," Talanta, vol. 77, no. 2, 447 pp. 710-717, 2008.

[11] E. Danesh et al., "Development of a new generation of Ammonia sen- 449 sors on printed polymeric hotplates," Anal. Chem., vol. 86, no. 18, 450 pp. 8951-8958, Aug. 2014.

[12] A. Falco, A. Rivadeneyra, F. C. Loghin, J. F. Salmeron, P. Lugli, 452 and A. Abdelhalim, "Towards low-power electronics: Self-recovering 453 and flexible gas sensors," J. Mater. Chem. A, vol. 6, pp. 7107-7113, 454 Mar. 2018.

[13] A. Abdellah, A. Yaqub, C. Ferrari, B. Fabel, P. Lugli, and G. Scarpa, 456 "Spray deposition of highly uniform CNT films and their application 457 in gas sensing," in Proc. 11th IEEE Int. Conf. Nanotechnol., 2011, 458 pp. $1118-1123$

[14] E. Contes-de Jesus, J. Li, and C. R. Cabrera, "Latest advances in mod- 460 ified/functionalized carbon nanotube- based gas sensors," in Syntheses 461 and Applications of Carbon Nanotubes and Their Composites. London, 462 U.K.: IntechOpen, 2013, pp. 337-366.

[15] A. Abdelhalim, A. Abdellah, G. Scarpa, and P. Lugli, "Fabrication of 464 carbon nanotube thin films on flexible substrates by spray deposition 465 and transfer printing," Carbon, vol. 61, pp. 72-79, Sep. 2013.

[16] A. Abdelhalim, M. Winkler, F. Loghin, C. Zeiser, P. Lugli, and 467 A. Abdellah, "Highly sensitive and selective carbon nanotube-based 468 gas sensor arrays functionalized with different metallic nanoparticles," 469 Sensors Actuators B Chem., vol. 220, pp. 1288-1296, Dec. 2015.

[17] A. Abdellah et al., "Flexible carbon nanotube based gas sensors fabri- 471 cated by large-scale spray deposition," IEEE Sensors J., vol. 13, no. 10, 472 pp. 4014-4021, Oct. 2013.

[18] A. Abdelhalim, A. Falco, F. Loghin, P. Lugli, J. F. Salmerón, and 474 A. Rivadeneyra, "Flexible NH3 sensor based on spray deposition and 475 inkjet printing," in Proc. IEEE SENSORS, 2016, pp. 1-3. 476

[19] S. Claramunt et al., "Flexible gas sensor array with an embedded 477 heater based on metal decorated carbon nanofibres," Sensors Actuators 478 B Chem., vol. 187, pp. 401-406, Oct. 2013.

[20] G. P. Evans, D. J. Buckley, N. T. Skipper, and I. P. Parkin, "Single-walled 480 carbon nanotube composite inks for printed gas sensors: Enhanced 481 detection of $\mathrm{NO} 2, \mathrm{NH} 3, \mathrm{EtOH}$ and acetone," $R S C A d v$, vol. 4, 482 pp. 51395-51403, Oct. 2014.

[21] J. H.-L. Lu and B. Jung, "Sensor conditioning circuits," in Integrated 48 Circuits for Analog Signal Processing. New York, NY, USA: Springer, 48 2013, pp. 223-242.

[22] C. K. Alexander and M. N. O. Sadiku, Fundamentals of Electric 487 Circuits. Boston, MA, USA: McGraw-Hill, 2009.

[23] P. Soderquist and M. Leeser, "An area/performance comparison of 489 subtractive and multiplicative divide/square root implementations," in 490 Proc. 12th IEEE Symp. Comput. Arithmetic, 1995, pp. 132-139. 491

[24] T. Drane, W.-C. Cheung, and G. A. Constantinides, "Correctly rounded 492 constant integer division via multiply-add," in Proc. IEEE Int. Symp. 493 Circuits Syst., 2012, pp. 1243-1246. 
495 [25] H. F. Ugurdag, A. Bayram, V. E. Levent, and S. Gören, "Efficient combinational circuits for division by small integer constants," in Proc. IEEE 23rd Symp. Comput. Arithmetic (ARITH), 2016, pp. 1-7.

498 [26] M. K. Muezzinoglu, "Acceleration of chemo-sensory information processing using transient features," Sensors Actuators B Chem., vol. 137, no. 2, pp. 507-512, Apr. 2009.

[27] D. Kumar, "Investigation of single wall nanotube gas sensor recovery behavior in the presence of UV," Adv. Mater. Lett., vol. 7, no. 4, pp. 262-266, Apr. 2016.

[28] G. Chen, T. M. Paronyan, E. M. Pigos, and A. R. Harutyunyan, "Enhanced gas sensing in pristine carbon nanotubes under continuous ultraviolet light illumination," Sci. Rep., vol. 2, p. 343, Mar. 2012.

[29] J. Li, Y. Lu, Q. Ye, M. Cinke, J. Han, and M. Meyyappan, "Carbon nanotube sensors for gas and organic vapor detection," Nano Lett., vol. 3 no. 7, pp. 929-933, Jul. 2003.

[30] W.-S. Cho, S.-I. Moon, K.-K. Paek, Y.-H. Lee, J.-H. Park, and B.-K. Ju, "Patterned multiwall carbon nanotube films as materials of $\mathrm{NO}_{2}$ gas sensors," Sensors Actuators B Chem., vol. 119, no. 1, pp. 180-185, Nov. 2006.

31] T. Ueda, M. M. H. Bhuiyan, H. Norimatsu, S. Katsuki, T. Ikegami, and F. Mitsugi, "Development of carbon nanotube-based gas sensors for $N O_{x}$ gas detection working at low temperature," Physica E Low Dimensional Syst. Nanostruct., vol. 40, no. 7, pp. 2272-2277, May 2008.

[32] S. I. Moon et al., "Bias-heating recovery of MWCNT gas sensor," Mater. Lett., vol. 62, no. 16, pp. 2422-2425, Jun. 2008.

[33] Spartan-6 FPGA Data Sheet: DC and Switching Characteristics (V3.1.1), Xilinx Inc., San Jose, CA, USA, Jan. 2015

[34] Cyclone II Device Handbook, vol. 1, Altera Corporat., San Jose, CA, USA, 2008.

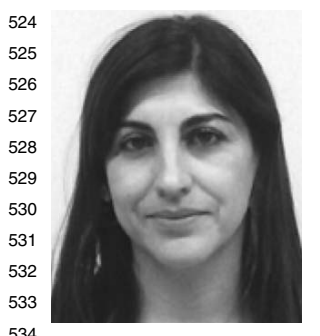

Encarnación Castillo received the M.Sc. and $\mathrm{Ph} . \mathrm{D}$. degrees in electronic engineering from the University of Granada, Granada, Spain, in 2002 and 2008, respectively.

From 2003 to 2005, she was a Research Fellow with the Department of Electronics and Computer Technology, University of Granada, where she is currently an Associate Professor. During her research fellowship, she carried out part of her research with the Department of Electrical and Computer Engineering, Florida State University, Tallahassee, 535 FL, USA. She has authored over 50 technical papers in international journals 536 and conferences. Her current research interests include the protection of IP 537 protection of very large-scale integration (VLSI) and field-programmable gate 538 arrays-based systems, as well as residue number system arithmetic, VLSI and 539 FPL signal processing systems, and the combination of digital and analog pro540 grammable technologies for smart instrumentation for biosignal processing. 541 Dr. Castillo also serves regularly as a reviewer for IEEE journals.

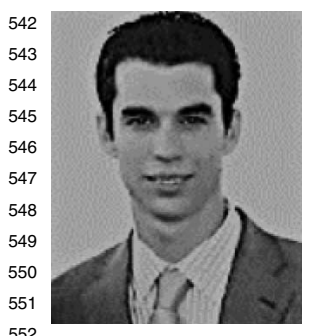

José F. Salmerón received the degrees in telecommunication engineering and electronics engineering from the University of Granada, Granada, Spain, in 2009 and 2011, respectively, and the master's degree in computer and network engineering and the Ph.D. degree in development of sensing capabilities in RFID technologies from the Technical University of Berlin, Berlin, Germany, in 2012 and 2014, respectively.

$\mathrm{He}$ is currently a Post-Doctoral Researcher with the Institute for Nanoelectronics, Technical 553 University of Munich, Munich, Germany, where he is involved in the design 554 and development of smart RFID labels with sensing capabilities.

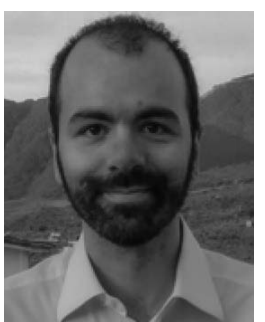

Aniello Falco received the B.Sc. and M.Sc. degrees 555 in electrical engineering from the University of 556 Salerno, Salerno, Italy, in 2010 and 2013, respec- 557 tively, and the Ph.D. degree from the Technical 558 University of Munich, Munich, Germany, under the 559 supervision of Prof. P. Lugli.

In 2013, he joined the Institute for 561 Nanoelectronics, Technical University of Munich, 562 where he conducted research on the solution 563 processing of nanomaterials, for biological and 564 sensing applications. Then, he moved to the Free 565 University of Bozen-Bolzano, Bolzano, Italy, where he collaborated with his 566 former supervisor to setup and develop a new research group on printed 567 electronics.

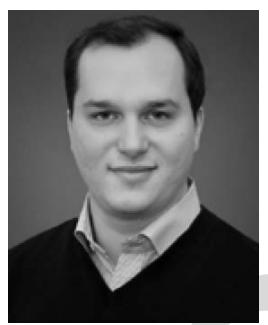

Florin C. Loghin received the M.Sc. degree in elec- 569 tronic engineering from the Technical University of 570 Munich, Munich, Germany, in 2015, where he is cur- 571 rently pursuing the Ph.D. degree with the Institute 572 for Nanoelectronics. 573

His current research interests include the devel- 574 oping of carbon nanotube-based thin-film transistors 575 and solution-processable-based organic electronics. 576

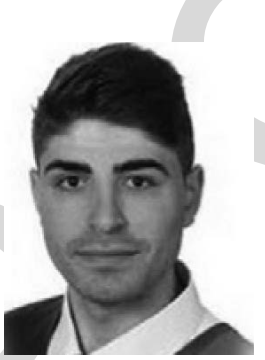

Francisco J. Romero received the B.Eng. degree 577 (with valedictorian mention) in telecommunication 578 engineering and the M.Eng. degree in telecommuni- 579 cations engineering from the University of Granada, 580 Granada, Spain, in 2016 and 2018, respectively, 581 where he is currently pursuing the Ph.D. degree with 582 a national predoctoral scholarship. $\quad 583$

In 2015, he joined the Department of Electronics 584 and Computer Technology, University of Granada as 585 an Ungraduated Researcher. His current research 586 interests include graphene-based sensors, flexible 587 electronics, and IoT embedded systems.

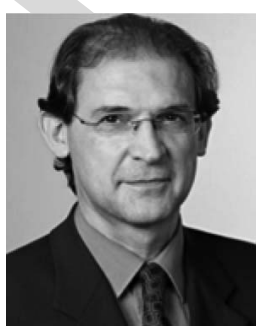

Paolo Lugli received the degree in physics from the 589 University of Modena, Modena, Italy, in 1979, and 590 the M.Sc. and Ph.D. degrees in electrical engineering 591 from Colorado State University, Fort Collins, CO, 592 USA, in 1982 and 1985, respectively. 593

$\mathrm{He}$ is currently the President of the Free 594 University of Bozen-Bolzano, Bolzano, Italy. He has 595 authored over 350 scientific papers.

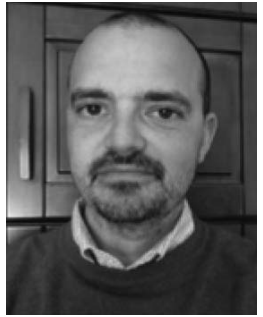

Diego P. Morales received the M.Sc. degree 597 in electronic engineering and the Ph.D. degree 598 in electronic engineering from the University 599 of Granada, Granada, Spain, in 2001 and 2011, 600 respectively.

$\mathrm{He}$ was an Associate Professor with the 602 Department of Computer Architecture and 603 Electronics, University of Almería, Almería, Spain. 604 $\mathrm{He}$ joined the Department of Electronics and 605 Computer Technology, University of Granada, 606 where he currently serves as a Tenured Professor. 607 His current research interest includes developing reconfigurable applications. 608

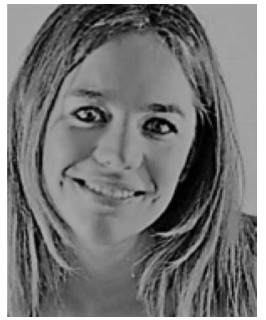

Almudena Rivadeneyra received the master's 609 AQ6 degrees in telecommunication engineering, environ- 610 mental sciences, and electronics engineering and the 611 $\mathrm{Ph} . \mathrm{D}$. degree in design and development of envi- 612 ronmental sensors from the University of Granada, 613 Granada, Spain, in 2009, 2009, 2012, and 2014, 614 respectively.

Since 2015, she has been with the Institute for 616 Nanoelectronics, Technical University of Munich, 617 Munich, Germany, where her research is centered in 618 printed and flexible electronics with a special focus 619 on sensors and RFID technology. 